\title{
Macro Dark Matter Self-gravitating Halos around Galaxies
}

\author{
Marco Merafina*† \\ "Sapienza" University of Rome, p.le A. Moro 5, I-00185 Rome (Italy) \\ E-mail: marco.merafinadromal.infn.it

\section{Francesco G. Saturni} \\ "Sapienza" University of Rome, p.le A. Moro 5, I-00185 Rome (Italy) \\ E-mail: Erancesco.gabriele.saturnieroma1.infn.it
}

\begin{abstract}
A new family of nonrelativistic, Newtonian non-quantum equilibrium configurations describing galactic halos is introduced taking into account a new possibility to identify particles with masses larger than $1 \mathrm{GeV}$ as components of the dark matter. This possibility may have important implications on the formation of very massive particles during the Big Bang. The obtained results are in agreement with the requested values in mass and radius in order to be consistent with the rotational velocity curve observed in the Galaxy. Additionally, the average density of such dark matter halos is similar to that derived for halos of dwarf spheroidal galaxies, which can therefore be interpreted as downscaled versions of larger dark matter distributions around Milky Way-sized galaxies and hint for a common origin of the two families of cosmic structures.
\end{abstract}

Frontier Research in Astrophysics - II

23-28 May 2016

Mondello (Palermo), Italy

\footnotetext{
*Speaker.

${ }^{\dagger}$ In collaboration with the Kaonnis Group at INFN-LNF (Rome, Italy).
} 


\section{Introduction}

Dark matter (DM) is one of the current challenges for modern astrophysics. Originally introduced in order to explain the flat rotation curves of spiral galaxies [四], DM is also required as a fundamental component ( $\sim 30 \%$; see e.g. [[] and refs. therein) of the Universe's energy content. Unfortunately, the DM weird physical property to do not couple with radiation as baryonic matter, required in order to explain its invisibility to traditional astronomical observations (e.g., [B] $)$, prevents astrophysicists to directly provide data on its constituents.

Several efforts have been made in order to identify plausible DM candidates, both on the side of elementary particles (see e.g. [四] for a review) and macroscopic objects (MACHOs; e.g., [[]]). However, without any direct hint about DM physics, the parameter space covered by the families of plausible DM candidates extends over many orders of magnitude of masses (for e.g. the elementary particles, ranging from $\sim 10^{-15} \mathrm{GeV}$ of axions up to $\sim 10^{15} \mathrm{GeV}$ of "wimpzillas") and cross sections (from $\sim 10^{-35} \mathrm{pb}$ of gravitinos up to $\sim 1 \mathrm{pb}$ of neutrinos).

In past years, a common idea of DM physics was that it shoud have been somewhat related to families of particles beyond the Standard Model (SM), such as those arising from supersymmetric theories (e.g., [6]). However, extensive runs performed at the Large Hadron Collider (LHC) in order to unveil traces of missing energy and momentum in baryonic collisions - a typical signature of events with production of non-interacting DM - have shown no clear features of such phenomena in large energy ranges, thus leading to a progressive exclusion of supersymmetric DM particles (e.g., [四]) although some possibilities still remain valid (e.g., [[]]). Similarly, the investigation of the density of MACHOs in the Milky Way and in extragalactic halos has shown that such objects are not abundant enough to represent a significant fraction of the DM mass (e.g., [Q] $)$.

Recently, a framework for the astronomical search of DM signals has been arising from the possibility that DM particles self-interact via annihilation or decay to produce SM pairs, that subsequently annihilate into final-state photons (e.g., [U], []]). If the mass of the DM candidates is sufficiently high (more than some $\mathrm{GeV}$ ), such photons could be detected on Earth through emission of Cherenkov radiation in the atmosphere, a task possibly at reach of next-generation Cherenkov telescope (e.g., [ए]2]).

In this paper, we present the new hypothesis of quark conglomerates with strangeness as basic DM components, and work out the expected macroscopic properties of the resulting DM halo around a Milky Way-sized spiral galaxy. We then compare the average properties of such a halo with the corresponding quantities derived from recent Fermi-LAT observations at $3 \sigma$ confidence level of $\gamma$-rays from the dwarf spheroidal galaxy (dSph) Reticulum II (RetII; [ए3]]), under the assumption that the $\gamma$-ray emission is due to DM self-interaction.

\section{Milky Way halo modelling}

Preliminarly, we can consider a very simplified model of spiral galaxy in order to roughly analyze the rotation curve to be compared with the observed one. This trivial model is spherically symmetric, composed by a central bulge with uniform density $\rho$ and a disk with a negligible mass; the mass is totally concentrated in the bulge. We have the following mass distribution 


$$
\begin{cases}M(r)=\frac{4}{3} \pi \rho r^{3} & \text { for } r \leq R_{b} \\ M(r)=M & \text { for } r>R_{b},\end{cases}
$$

where $R_{b}$ is the bulge radius. Using the general definition of rotation velocity

$$
v(r)=\sqrt{\frac{G M(r)}{r}}
$$

we obtain the following expression

$$
\left\{\begin{array}{lll}
v(r)=\sqrt{\frac{4}{3} \pi G \rho} r & \text { for } r \leq R_{b} & \text { (rigidly rotating body) } \\
v(r)=\sqrt{G M / r} & \text { for } r>R_{b} & \text { (Keplerian velocity) }
\end{array}\right.
$$

By comparing the rotation velocity curve with observative data, it becomes clear the difference at large values of $r$. Theoretical results give a decreasing Keplerian prediction, whereas observative data show a flat behavior around $200 \mathrm{Km} / \mathrm{s}$ (see Fig. W).

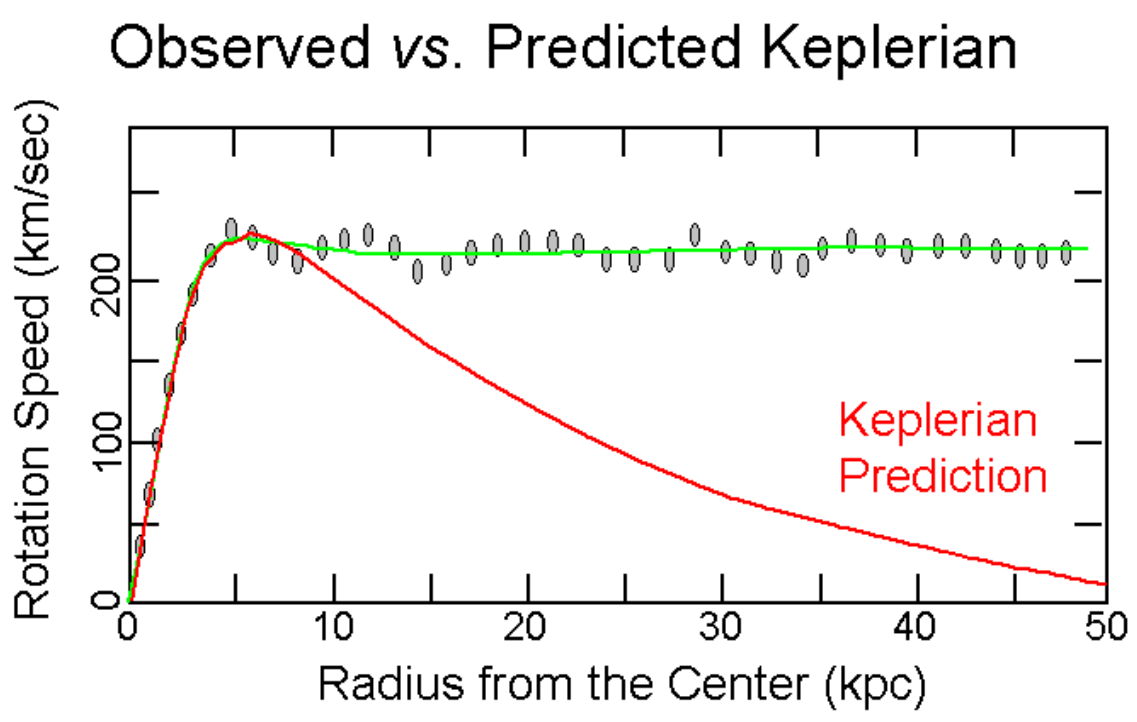

Figure 1: Rotation curve in spiral galaxies and predicted behavior.

Only the existence of a Galactic halo composed by DM, like the one first introduced by [W], can explain this observative behavior. Subsequently, more accurate observations have been performed and the flat behavior has been confirmed (see Fig. 『), resulting in agreement with the existence of a halo of mass $M_{\text {halo }} \sim 10 M_{\text {gal }}$ and radius $R_{\text {halo }} \sim 10 R_{\text {gal }}$.

Some questions arise from this preliminary analysis. What is the nature of the DM? What is the particle composition of the halo? What is the mass of these particles? The problem was widely discussed since 1970s, and the construction of DM halos models has experienced a significant development, with the hypothesis of a massive neutrino (with mass of the order of a few tens of $\mathrm{eV}$ ), generically named weakly-interacting massive particle (WIMP), as a diffuse component, due to the importance of beta decay in the stellar evolution (see e.g. [4, [15, [16]). 


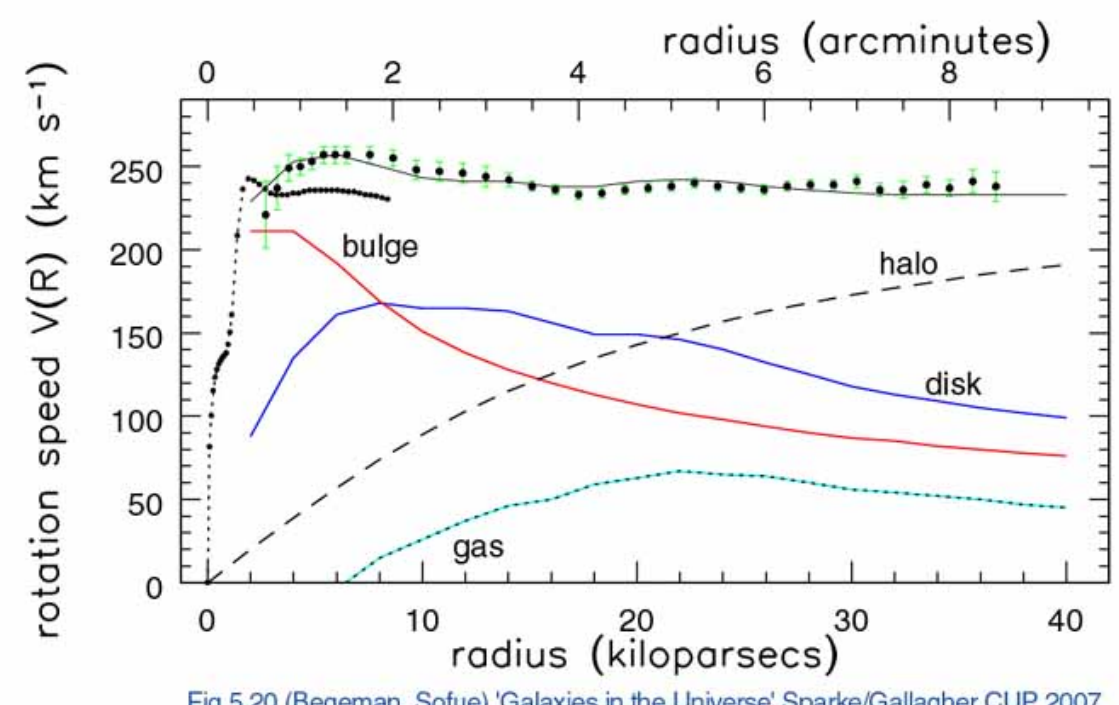

Figure 2: Different components in our Galaxy (spiral) and contribution to the rotation curve.

The equilibrium of such a self-gravitating halo can be solved by considering a degenerate Fermi gas of neutrinos, and using a polytropic model with $n=3 / 2$. The halo mass and radius are straightforward to obtain, their expressions given by

$$
\begin{aligned}
& M=\frac{3}{2}\left(\frac{\pi}{2}\right)^{3 / 2}(2.71406) \frac{\hbar^{3}}{G^{3 / 2} m_{v}^{4}} \rho_{0}^{1 / 2} \\
& R=\frac{(9 \pi)^{1 / 6}}{2 \sqrt{2}}(3.65375) \frac{\hbar}{G^{1 / 2} m_{v}^{4 / 3}} \rho_{0}^{-1 / 6}
\end{aligned}
$$

where $m_{v}$ is the neutrino rest mass and $\rho_{0}$ the central density. Introducing the above conditions $M_{\text {halo }} \sim 10 M_{\text {gal }}$ and $R_{\text {halo }} \sim 10 R_{\text {gal }}$ implies a central density $\rho_{0} \sim 10^{-25} \mathrm{~g} \mathrm{~cm}^{-3}$ for a neutrino rest mass $m_{v} \sim 10 \mathrm{eV}$. Moreover, combining Eqs. $[2.4$ and $[2.5$ leads to a simple relation between mass and radius of our Galaxy

$$
R \simeq 90\left(\frac{M}{10^{12} M_{\odot}}\right)^{-1 / 3} \mathrm{kpc}
$$

A non-relativistic treatment of the halo equilibrium is clearly the most appropriate given that both the critical density $\rho_{c r}$ and the general relativity factor $G M / R c^{2}$ are small, i.e.

$$
\rho_{c r}=\frac{m_{v}^{4} c^{3}}{3 \pi^{2} \hbar^{3}}=7.8 \cdot 10^{-17} \mathrm{~g} \mathrm{~cm}^{-3} \gg \rho_{0}
$$

and

$$
\frac{G M}{R c^{2}}=4.8 \cdot 10^{-7} \ll 1
$$




\section{Strange dark matter halos}

The WIMP hypothesis is not unique in the framework of possible dark matter particle candidates. There are in fact a lot more candidates (fuzzy DM, hidden photons, ultra-light axions...) discussed in the literature (see e.g. [䧃]), with $m_{D M}$ in principle anywhere between $10^{-31} \mathrm{GeV}$ and $10^{18} \mathrm{GeV}$ (see Fig. B]).

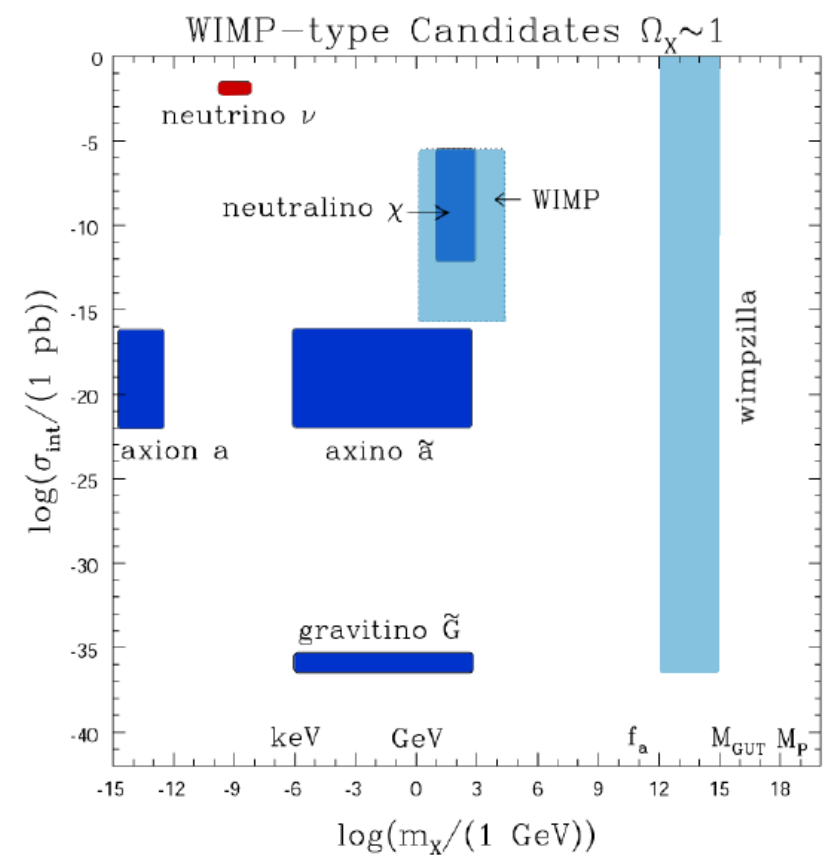

Figure 3: A partial review of different DM candidates (particles only; adapted from [四]).

Furthermore, the possibility to have non-WIMP DM canditates can be taken into account. This alternative and fascinating hypothesis involves strange massive particles (SMPs; $m c^{2} \sim 1$ $\mathrm{GeV}$ ) directly produced in the framework of the Big Bang standard model. Such a scenario arises by the simple consideration that the interaction rate between baryons and DM particles may be suppressed if DM particles are produced with large mass and consequently low number density. In fact, this rate is proportional to $n \sigma v$, with $n$ the number density, $\sigma$ the cross section and $v$ the particle velocity. Therefore, DM particles with low effective interaction rate (even for large cross sections) should evolve independently as massive Big-Bang relics, constituting a useful background in the formation of galactic halos. Among different possible SMP candidates for DM, particles with strangeness may play a very interesting role.

In order to better describe this hypothesis, it is necessary to individuate stable particles with sufficiently large lifetime. One possiblity is given by a recent paper introducing $\Lambda^{*}$ matter as possible constituents of neutron stars and DM [107]. In spite of the large mass of the $s$ quark with respect to $u$ and $d$ quarks, the chemical potential due to the Pauli exclusion principle should favour stable quark configurations (strange quark matter conglomerates) with the same (approximate) number of $u, d$ and $s$ quarks. 
One possibility among different $\bar{K} N$ states may be $\Lambda^{*}(1405) \equiv K^{-} p$. As a single particle, it undergoes to strong decay and is therefore highly ustable. Differently, it may become stable in an aggregated state (see Fig. $⿴$ ), forming a $\Lambda^{*}$ conglomerate which can be considered as a macroparticle with a rest mass $m^{*}<N m_{\Lambda^{*}} \sim \mathrm{GeV}$, where $N \sim 10$ or less.
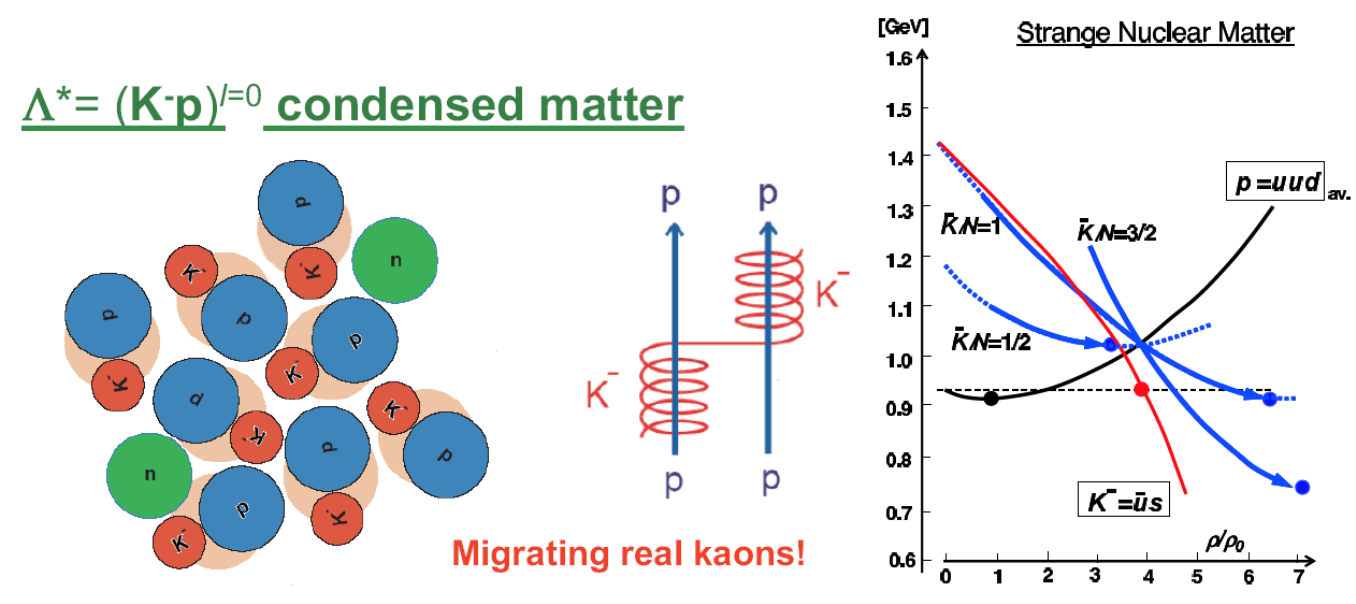

Figure 4: Left: speculated $K^{-} p=\Lambda^{*}$ matter with a quasi- $\Lambda^{*}$ as an atomic constituent, where $K^{-}$migrate among protons, producing high-density kaonic matter. Right: speculated diagrams for the density dependency of the bound-state energies of various nuclear composite systems $\left(p K^{-}\right)^{m} n^{n}$. Both the $\bar{K} N$ energy (red curve) and the nuclear compression (black curve) are shown. The total energies for representative fractions of $K^{-} / N(=1 / 2,1,3 / 2)$ are depicted by respective blue curves, showing minima at high density and low energy. Density-dependent enhanced $\bar{K} N$ interactions are assumed (figure in Akaishi \& Yamazaki [[]]).

Although the large densities in the central regions of the neutron stars may suggest the production of processes $\bar{K}^{0} n \rightarrow K^{-} p$ for the formation of hyperon cores, due to the value of the required critical density (of the order of $10^{19} \mathrm{~g} \mathrm{~cm}^{-3}$ ) we stress that this hypothesis is more favourable in the cosmological field, where the possibility to have arbitrarily high values of density and temperature are not precluded. In this framework, the formation of ultra-dense kaonic nuclear states as a partial constituent of DM is more realistic. Moreover, while in the current experiments for $\Lambda^{*}$ production one obtains a combination of $\sim 90 \%$ of $\bar{K} N$ (resonance) and only $\sim 10 \%$ of $\Sigma \pi$ (the true particle), during the Big Bang only the particle $\Sigma \pi$ was formed at such high densities.

We can thus hypothesize the following scenario. During the first phase of the Big Bang, at sufficiently high density (and temperature), the conditions for the formation of $\Lambda^{*}$ particles are set. With the formation of conglomerates, the negative contribution of the binding energy due to strong interactions reduces the effective mass of $\Lambda^{*}$ from $1405 \mathrm{MeV}$ to a value lower enough $(\sim 1200$ $\mathrm{MeV}$; Curceanu 2015, private communication) for closing the main allowed decay channels. In this way, the $\Lambda^{*}$ conglomerates become stable, and can be considered as a single particle of mass $m^{*} \sim 5 \div 10 \mathrm{GeV}$ with a very low probability to interact with baryonic matter. These Big-Bang relic particles can form galactic halos.

This scenario must clearly be considered as only a possible hypothesis of formation of DM, and its further investigation is needed, especially from the quantitative point of view. One of the problems is related to the expansion rate of the Universe: if cooling rate and decrease of density are 
in fact faster than the stabilization rate of conglomerates, the process is not implemented. Another problem is connected with the collisions among conglomerates: fluctuations of density with respect to the average value may increase the collision rate and thus create the conditions for instability of such systems. These particular conditions can also be reached in the central regions of a single galactic halo, if the central density of visible matter (galaxy) and the gravitational field are high enough to increase the probability of collision among conglomerates. During the collisions, kinetic energy can give the particles of a single conglomerate enough energy to reach a new instability, and then decay in standard model pairs that subsequently annihilate in $\gamma$-ray photons. Therefore, it is important to look into high-density regions, where the collisions are more probable, in order to obtain evidences of DM existence through the indirect detection of gamma rays from DM selfinteraction.

In order to calculate self-gravitating equilibrium configurations of DM halos, we now explore the possibility of having halos composed by stable $\Lambda^{*}$ conglomerates, although their existence is still debated. Despite we expect a high density $\rho>10^{15} \mathrm{~g} \mathrm{~cm}^{-3}$ in the internal structure of the conglomerate, it is not relevant for our purposes. Therefore, we consider the single $\Lambda^{*}$ conglomerate like a massive particle of mass $m^{*}$ interacting only gravitationally with the other conglomerates composing the halo.

First, we consider a semi-degenerate gas of particles with a rest mass $m^{*}=5 \div 10 \mathrm{GeV}$. We look for halos with mass $M \sim 10^{12} M_{\odot}$ and radius $R \sim 100 \mathrm{kpc}$, with a mean density $\bar{\rho}$ of the order of $10^{-26} \mathrm{~g} \mathrm{~cm}^{-3}$. For $m^{*}=5 \mathrm{GeV}$ we obtain

$$
\rho_{c r}=\frac{m^{* 4} c^{3}}{3 \pi^{2} \hbar^{3}}=4.9 \cdot 10^{18} \mathrm{~g} \mathrm{~cm}^{-3} \gg \bar{\rho}
$$

and

$$
\frac{G M}{R c^{2}}=4.8 \cdot 10^{-7} \ll 1
$$

This demonstrates that also strange DM halos are non-relativistic and Newtonian.

For the equilibrium configuration, we consider a semi-degenerate Fermi distribution function with a cutoff in energy given by the following expression [एष]

$$
\begin{cases}f(\varepsilon)=\frac{g}{h^{3}}\left[\frac{1-e^{\left(\varepsilon-\varepsilon_{c}\right) / k T}}{e^{(\varepsilon-\mu) / k T}+1}\right] & \text { for } \varepsilon \leq \varepsilon_{c} \\ f(\varepsilon)=0 & \text { for } \varepsilon>\varepsilon_{c},\end{cases}
$$

where $\varepsilon_{c}=m\left(\varphi_{R}-\varphi\right)$ is the cutoff energy, $\varphi$ is the gravitational potential, $\mu$ is the chemical potential and $g=2 s+1$ is the multiplicity of quantum states. The mass density $\rho$ is given by

$$
\rho=m \int f(\varepsilon) d^{3} q .
$$

For the gravitational equilibrium, we use the Poisson equation

$$
\frac{1}{r^{2}} \frac{d}{d r}\left(r^{2} \frac{d \varphi}{d r}\right)=4 \pi G \rho \text { with } \varphi^{\prime}(0)=0 ; \varphi(0)=\varphi_{0} .
$$

By integrating Eq. B.5, we obtain different equilibrium configurations at different values of $W_{0}$ and $\theta_{R}$, where $W_{0}$ is the value of $W=\varepsilon_{c} / k T$ at the center of the configuration and $\theta_{R}$ is the value 
of $\theta=\mu / k T$ at the border of the configuration. These quantities are related through the relation $\theta_{R}=\theta-W \leq 0$ [ㅍ, [2]]. The solutions also depend on $m$ (mass of the particle) and $\sigma$ (surface velocity dispersion) through scaling laws. The results are summarized in diagrams of $M$ versus $\rho_{0}$ and $R$ versus $\rho_{0}$ for $m=5 \mathrm{GeV}$ and $\sigma=400 \mathrm{~km} \mathrm{~s}^{-1}$ (see Fig. (1).
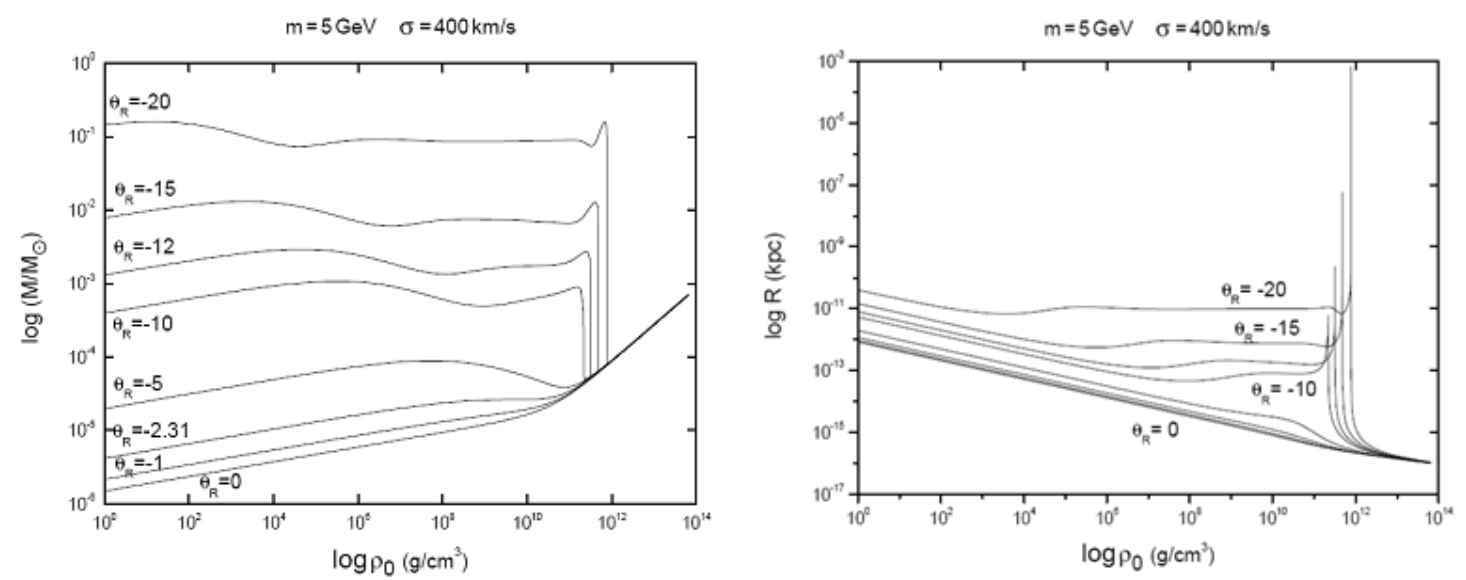

Figure 5: Equilibrium configurations at different values of $\theta_{R}$. A phase transition from non-quantum to fully degenerate configurations at densities around $10^{12} \mathrm{~g} \mathrm{~cm}^{-3}$ is clearly visible.

It is clear that the particle mass value $m^{*}=5 \mathrm{GeV}$ does not allow to obtain the expected values of central density, mass and radius for a galactic halo. In fact, we have $\rho_{0} \propto \sigma^{3} m^{4}, M \propto$ $\sigma^{3 / 2} m^{-2}$ and $R \propto \sigma^{-1 / 2} m^{-2}$. This results in too large densities, and too small masses and radii, implying that the semi-degenerate regime is not appropriate to describe strange DM halos. We need much more negative values of $\theta_{R}$, typical of a classical regime well described by the Boltzmann (King) distribution function with cutoff in energy. Therefore, strange DM halos are non-relativistic, Newtonian and do not follow quantum statistics.

In order to obtain halos with appropriate densities, masses and radii, we calculate equilibrium configurations at fixed central density $\left(\rho_{0}=10^{-24} \mathrm{~g} \mathrm{~cm}^{-3}\right)$ and particle mass $\left(m^{*}=5 \mathrm{GeV}\right)$, while increasing the value of $-\theta_{R}$ until we reach $M \sim 10^{12} M_{\odot}$ and $R \sim 100 \mathrm{kpc}$ (see Fig. G). We compute solutions in the range $W_{0}=1 \div 10$ (for globular clusters, the most significant values are between 4 and 8; for galactic halos we expect even less). In this regime, the dependence on $\theta_{R}$ become a scaling law. It is possible to make a tuning by varying the central density $\rho_{0}$ and the parameter $\theta_{R}$ in order to match the requested values in $M$ and $R$, also at different values of $W_{0}$. The obtained results for $m^{*}=5 \mathrm{GeV}$ and $\rho_{0}=10^{-24} \mathrm{~g} / \mathrm{cm}^{3}$ are very satisfying: we obtain $\theta_{R}=-79$ and $W_{0}=1.9$, implying a halo mass $M=9.78 \cdot 10^{11} M_{\odot}$, a halo radius $R=90.17 \mathrm{kpc}$, a mean halo density $\bar{\rho}=3 M / 4 \pi R^{3}=2.16 \cdot 10^{-26} \mathrm{~g} \mathrm{~cm}^{-3}$ and a velocity dispersion $\sigma_{v}=391 \mathrm{~km} \mathrm{~s}^{-1}$. The other solutions can be obtained from scaling laws involving the total mass $M$ and the radius $R$. We obtain

$$
\begin{gathered}
M=9.78 \cdot 10^{11}\left(\frac{\rho_{0}}{10^{-24} \mathrm{~g} \mathrm{~cm}^{-3}}\right)^{-1 / 2}\left(\frac{m^{*}}{5 \mathrm{GeV}}\right)^{-4} \mathrm{M}_{\odot} \\
R=90.17\left(\frac{\rho_{0}}{10^{-24} \mathrm{~g} \mathrm{~cm}^{-3}}\right)^{-1 / 6}\left(\frac{m^{*}}{5 \mathrm{GeV}}\right)^{-4 / 3} \mathrm{kpc}
\end{gathered}
$$


These results are summarized in a visual way in Fig. $\square$.

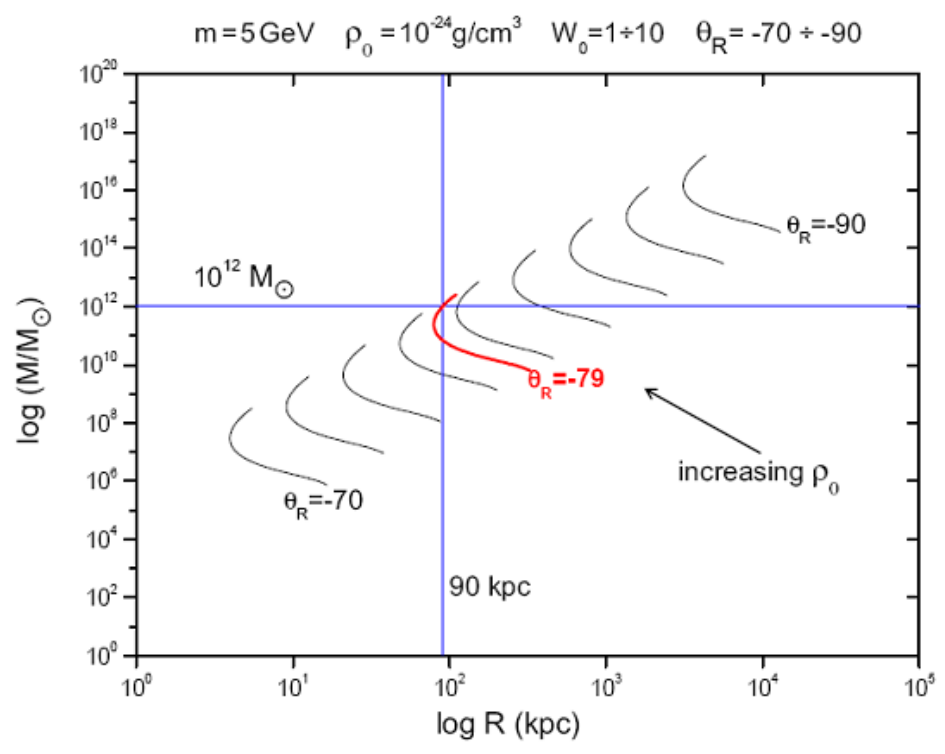

Figure 6: Individuation of the value of $\theta_{R}$ compatible with the requested values of mass and radius for spiral-galaxy halos. The position of a MW-sized halo (blue lines) is highlighted, along with the relevant value of $\theta_{R}$ (red curve).

\section{Comparison with dwarf galaxy halo properties}

The theoretical scenario presented in Sec. B, though fascinating, is deeply related to the existence of unobserved strange conglomerates; furthermore, the derived physical parameters of the DM halo hold in principle only for MW-sized spiral galaxies. In this section, we show how the average density of a strange DM halo is (possibly) common also to halos of different size like those surrounding the $\mathrm{dSphs}$, probably the most DM dominated objects in the local Universe, and that such halos can be obtained by scaling down the typical masses and radii for halos around normal galaxies. In order to do so, we derive the amount of DM in the dSph RetII by analyzing the kinematics of its member stars.

Discovered in 2015 by [ [D] in first-year Dark Energy Survey (DES) data, RetII is a faint MW satellite from which Fermi-LAT has detected an excess of gamma rays between $\sim 3$ and $\sim 10 \mathrm{GeV}$ [ㅍ] (see Fig. [). Such an excess is compatible with a flux coming from DM annihilation of particles with $m_{\chi} \sim 25 \mathrm{GeV}$ at $3 \sigma$ confidence level ${ }^{1}$; this value of $m_{\chi}$ is particularly interesting in the framework of DM made by conglomerates of $\Lambda^{*}$, since it is an integer multiple of the conglomerate mass $m^{*}=5 \mathrm{GeV}$ assumed in Sec. [1]. Under this hypothesis, this signal can therefore be used to constrain the amount of DM in RetII, i.e. its astrophysical factor for DM annihilation $J$ (e.g., [ए]]). We get

\footnotetext{
${ }^{1}$ Another tantalizing $\gamma$-ray detection is reported for the Galactic center by [ㄹ2]; however, its interpretation as a product of DM self-interaction with $m^{*} \sim 45 \mathrm{GeV}$ is still controversial.
} 


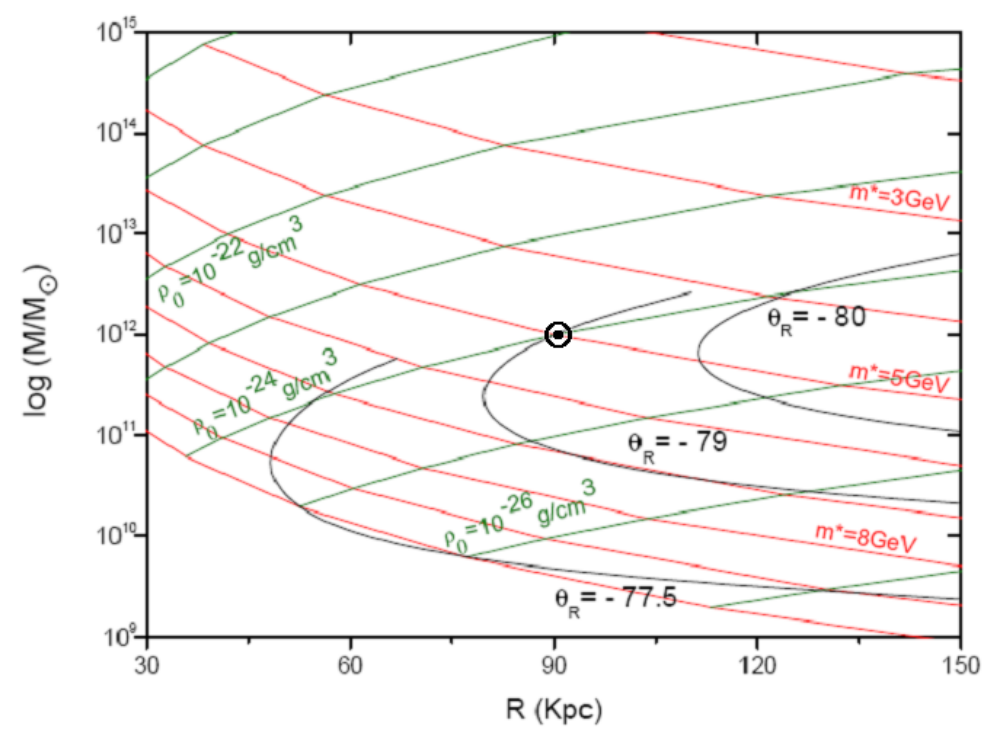

Figure 7: Solutions for MW-sized DM halos obtained through scaling laws between halo physical parameters and conglomerate masses. The relations between halo mass and size for different conglomerate masses (red curves), central DM densities (green curves) and concentration parameters (black curves) are plotted. The position of the MW-sized halo for $m^{*}=5 \mathrm{GeV}$ (open circle with dot) is also marked.

$$
J=\int_{\Delta \Omega} d \Omega \int_{l o s} \rho^{2}(s, \Omega) d s .
$$

Assuming an appropriate value for the velocity-averaged cross section of the annihilation process $\langle\sigma v\rangle \lesssim 2.2 \cdot 10^{-26} \mathrm{~cm}^{3} \mathrm{~s}^{-1}$ [23], one gets $J \gtrsim 4 \cdot 10^{19} \mathrm{GeV}^{2} \mathrm{~cm}^{-5}$ over a region of interest (ROI) of $0.5 \mathrm{deg}$ around RetII center.
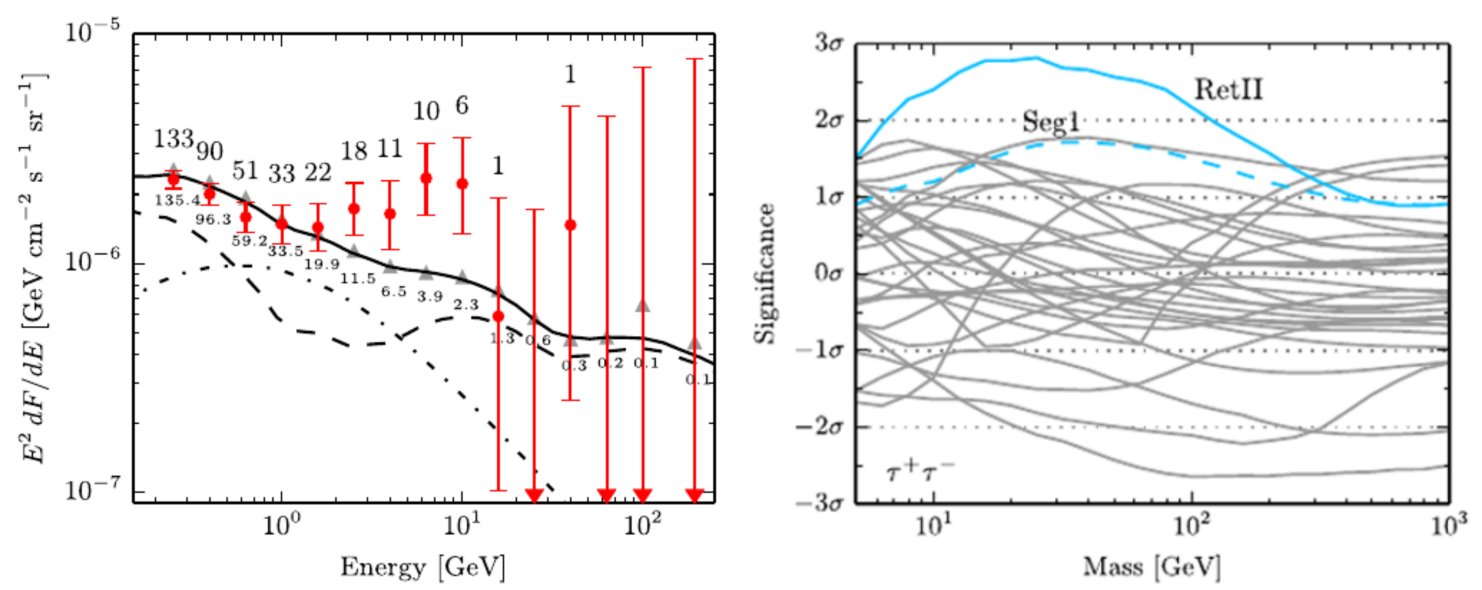

Figure 8: Fermi-LAT $\gamma$-ray flux excess and its statistical significance (adapted from [ए3]]). Left panel: energy spectrum from a ROI of 0.5 deg around the RetII center. Right panel: model-independent significance for DM annihilation into $\tau^{+} \tau^{-}$pairs.

Since the astrophysical factor for DM annihilation is defined as the integral of the squared DM 
density in the ROI along the line of sight, one can derive the rms value $\rho_{r m s}$ of this density from the measurement of $J$. We obtain

$$
J=\frac{\int_{\Delta \Omega} d \Omega \int_{l o s} \rho^{2}(s, \Omega) d s}{\int_{l o s} d s} \int_{l o s} d s=\left\langle\rho^{2}\right\rangle_{\Delta \Omega} D_{\odot} \Rightarrow \rho_{r m s}=\sqrt{\frac{J}{D_{\odot}}} .
$$

From Eq. 4.2, with $D_{\odot}=30 \pm 2 \mathrm{kpc}$ [2], [23] one obtains $\rho_{r m s}=\left(3.7_{-2.1}^{+8.4}\right) \cdot 10^{-26} \mathrm{~g} \mathrm{~cm}^{-3}$ as an estimate of the mean DM density of RetII halo, in excellent agreement with the theoretical value derived in Sec. [3 for MW-sized halos. One can argue that the belonging of RetII to the MW system naturally implies similar mean DM densities among gravitationally bound halos in (approximate) dynamical equilibrium; nonetheless, this is not necessarily true, if for instance the original distribution of DM halo parameters had implied very different scale densities for halos of different size.

A more reliable estimate of the DM halo parameters for RetII can be performed by repeating the Jeans analysis presented in [B]], i.e. integrating the moments of the phase-space distribution function for a steady-state, spherically symmetric and negligibly rotating collisionless system to obtain the second-order Jeans equation [25]:

$$
\frac{1}{n(r)}\left[\frac{d}{d r}\left(n \bar{v}_{r}^{2}\right)\right]+2 \frac{\beta_{\text {ani }}(r)}{r} \bar{v}_{r}^{2}(r)=-\frac{4 \pi G}{r^{2}} \int_{0}^{r} \rho_{D M}(s) s^{2} d s .
$$

Here, $n(r), \bar{v}_{r}^{2}(r)$ and $\beta_{a n i}(r)$ are the stellar number density, velocity dispersion and velocity anisotropy respectively. For the case of $\mathrm{dSphs}$, the solution to Eq. 4.31 relates the internal proper motions of stars to the amount of DM in the dSph halo, although only line-of-sight observables like the projected radius $R$, the surface brightness $\Sigma(R)$ and the projected stellar velocity dispersion $\sigma_{p}(R)$ can be used, namely

$$
\sigma_{p}^{2}(R)=\frac{2}{\Sigma(R)} \int_{R}^{+\infty}\left[1-\beta_{\text {ani }}(r)\left(\frac{R}{r}\right)^{2}\right] \frac{n(r) \bar{v}_{r}^{2}(r)}{\sqrt{r^{2}-R^{2}}} d r .
$$

In order to determine the parameters that best reproduce the observed properties of RetII, we run a simulation of $8 \cdot 10^{4}$ MCMC points with the CLUMPY ${ }^{2}$ software [26, [27] on the member stars of RetII [20], according to the prescriptions listed in [20, [0] ] and assuming an Einasto profile [B]] for the DM distribution

$$
\rho(r)=\rho_{s} e^{-\frac{2}{\alpha}\left[\left(\frac{r}{r_{s}}\right)^{\alpha}-1\right]} .
$$

The stellar number density $n(r)$ is preventively calculated by fitting a 3D Zhao-Hernquist profile [32, [33] to publicly available 2D photometric data of RetII [24], and the resulting parameters are used as a fixed input for CLUMPY. We take the stellar-kinematics data from [B4], estimating the membership probability $P_{i}$ of the $i$-th star with an estimation-of-membership (EM) algorithm [B5] and keeping only stars for which $P_{i} \geq 0.95$. In doing so, we find that an astrophysical factor $\log J=19.3_{-0.7}^{+1.1}$ at an integration angle of $0.5 \mathrm{deg}$, compatible at $1 \sigma$ level with the upper limit estimated by [113], is obtained from the assumed DM density profile with

\footnotetext{
${ }^{2}$ Available at https://lpsc.in2p3. fr/clumpy/.
} 


$$
\left\{\begin{array}{l}
\rho_{s}=\left(5.9_{-4.8}^{+21.0}\right) \cdot 10^{7} M_{\odot} \mathrm{kpc}^{-3} \\
r_{s}=0.52_{-0.50}^{+3.29} \mathrm{kpc} \\
\alpha=0.51_{-0.26}^{+0.35} .
\end{array}\right.
$$

For the purposes of calculating (i) the astrophysical factor for DM annihilation over a ROI of 0.5 deg diameter, and (ii) the average DM density in RetII halo, we integrate the DM density profile obtained from the MCMC run up to the tidal radius of the dSph halo. Using eq. 18 of [BO] we compute a value of $3.2 \mathrm{kpc}$ for $r_{t}$. In Fig. Q Q we show the computed projected stellar velocity dispersion $\sigma_{p}(R)$ and the astrophysical factor $J\left(\alpha_{i n t}\right)$ as a function of the projected radius $R$ from the dSph center and the integration angle $\alpha_{i n t}$ respectively. The computed $\sigma_{p}(R)$ is also visually compared with the corresponding measurements from [34], showing a very good agreement over the whole sampled range of projected radii.
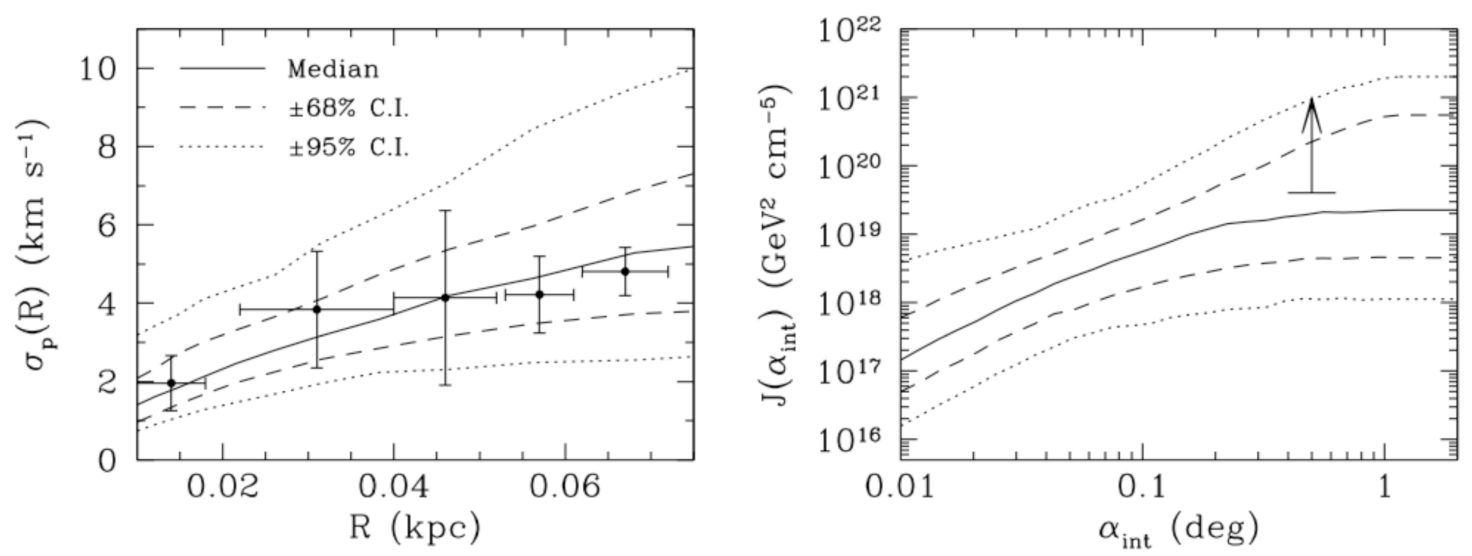

Figure 9: Left panel: RetII stellar velocity dispersion as a function of the projected radius from the dSph center. For comparison, the binned measurements from [B4] (dots) are overplotted together with their $1 \sigma$ errors computed over the identified member stars. Right panel: RetII astrophysical factor for DM annihilation as a function of the instrumental integration angle. The lower limit on $J\left(\alpha_{i n t}\right)$ derived by [[]] is reported as a visual confirmation of the goodness of the MCMC calculations. In both panels, median quantities (solid lines) are shown together with the corresponding confidence intervals at $68 \%$ (dashed lines) and 95\% probability (dotted lines).

Thus, the mean DM density $\left\langle\rho_{D M}\right\rangle$ over the volume enclosed within $r_{t}$ around the dSph center is given by

$$
\left\langle\rho_{D M}\right\rangle=\frac{4 \pi}{\mathscr{V}} \int_{0}^{r_{t}} \rho_{D M}(r) r^{2} d r
$$

with $\mathscr{V}=4 \pi r_{t}^{3} / 3$ (note that we are here neglecting any effect of a halo triaxiality; see e.g. [36]).

Using the parameters listed in Eq. 4.6, we get $\left\langle\rho_{D M}\right\rangle=\left(22.6_{-2.4}^{+9.6}\right) \cdot 10^{-26} \mathrm{~g} \mathrm{~cm}^{-3}$. This value is a factor of $\sim 6$ larger than the estimate of $\rho_{r m s}$ obtained from $J$, and an order of magnitude larger than the value for MW-sized DM halos constructed with strange conglomerates; however, the impact of stellar feedback, triaxiality and tidal interactions on the dynamical status of dSph DM halos is still largely unknown, meaning that at least the RetII mean density is overestimated. 
Therefore, a difference of roughly an order of magnitude between the mean densities of MW-sized and dSph-sized halos is acceptable in this framework to stress anyway a common origin for the two families of galactic structures. In Fig. 10 we show the scaling relation between the RetII halo parameters obtained from the Jeans analysis and the MW parameters for the theoretical SMP halo in a graphical way.

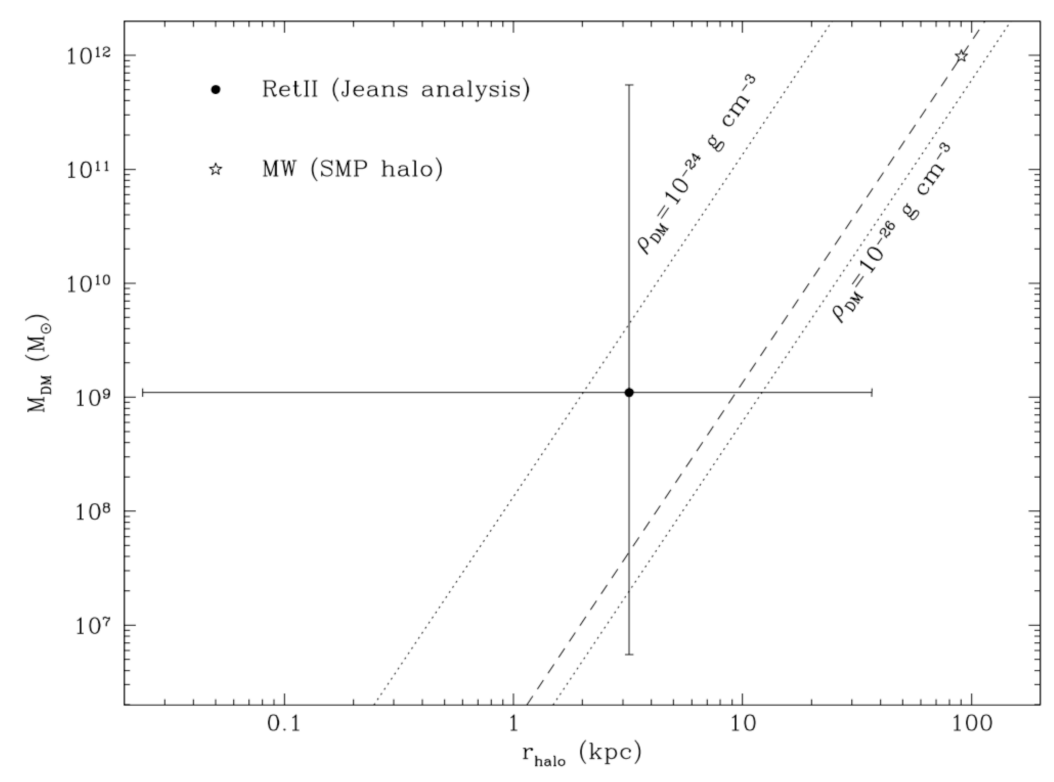

Figure 10: Scaling relation between RetII dSph DM halo parameters obtained from the Jeans analysis performed with the CLUMPY software (filled dot) and MW parameters for the theoretical DM halo constructed with SMPs (open star). The errors at 68\% confidence level are associated to the RetII measurements of halo mass and radius. For comparison, the relation $M_{D M} \propto r_{\text {halo }}^{3}$ at constant density $\rho_{D M}=2.2 \cdot 10^{-26} \mathrm{~g} \mathrm{~cm}^{-3}$ (dashed line) is reported, along with the same relation scaled at $10^{-24}$ and $10^{-26} \mathrm{~g} \mathrm{~cm}^{-3}$ (dotted lines).

\section{Conclusions}

In this paper, we proposed a possible scenario for DM origin in the Universe based on conglomerates of $\Lambda^{*}(1405)$ particles. These conglomerates form in the very early phases after the Big Bang, when the conditions of extreme density $\left(\rho \gg 10^{19} \mathrm{~g} \mathrm{~cm}^{-3}\right)$ and temperature may favor the aggregation of strange baryonic matter in stable structures that interact only gravitationally with ordinary matter; subsequently, when the Universe expands and cools down, the conglomerates formed in this way settle into galactic halos as "relic" DM.

We showed how the assumption of conglomerates with mass of $5 \mathrm{GeV}$ can lead to a good reproduction of the physical properties (mass, radius, concentration) of a typical MW-sized DM halo. Performing a Jeans analysis on the kinematical properties of the RetII dSph member stars, we also showed how the average DM density in halos of very different size is approximately maintained, hinting for a common origin of both families of structures.

As a final remark, we recommend to adopt some caution when considering the results presented here. In fact, the proposed scenario for DM composed by conglomerates of particles with 
strangeness is still tentative; at present, no quantitative models able to compute the stability and formation rate of strange conglomerates exist. Furthermore, the detection of $\gamma$-ray signals from DM halos is still controversial, and future observations with next-generation telescopes (such as the Cherenkov Telescope Array, $\mathrm{CTA}^{3}$ ) are needed in order to finally detect sources of $\gamma$-rays produced by DM self-interaction.

\section{References}

[1] Zwicky, F. 1933, Helvetica Physica Acta, 6, 110

[2] Planck Collaboration, Ade, P. A. R., Aghanim, N. et al. 2014, A\&A, 571, A16

[3] Kamionkowski, M. 2011, Visions of Discovery (Cambridge University Press), p. 247

[4] Roszkowski, L. 2004, Pramana, 62, 389

[5] Tisserand, P., Le Guillou, L., Afonso, C. et al. 2007, A\&A, 469, 387

[6] Jungman, G., Kamionkowski, M. \& Griest, K. 1996, Physics Reports, 267, 195

[7] Bechtle, P., Bringmann, T., Desch, K. et al. 2012, JHEP, 6, 98

[8] Cahill-Rowley, M., Hewett, J. L., Ismail, A. et al. 2015, Phys. Rev. D, 91, 055002

[9] Jaiyul, Y., Chanamé, J. \& Gould, A. 2004, ApJ, 601, 311

[10] Cembranos, J. A. R., de la Cruz-Dombriz, A., Dobado, A. et al. 2011, Journal of Physics Conference Series, 314,1

[11] Cirelli, M., Gaggero, D., Giesen, G. et al. 2014, JCAP, 12, 045

[12] Doro, M., Conrad, J., Emmanoulopoulos, D. et al. 2013, Astroparticle Physics, 43, 189

[13] Geringer-Sameth, A., Walker, M. G., Koushiappas, S. M. et al. 2015, Physical Review Letters, 115, 8

[14] Cowsik, R. \& McClelland, J. 1972, Phys. Rev. Lett., 29, 669

[15] Cowsik, R. \& McClelland, J. 1973, ApJ, 180, 7

[16] Tremaine, S. \& Gunn, J. E. 1979, Phys. Rev. Lett., 42, 407

[17] Akaishi, Y. \& Yamazaki, T. 2015, RIKEN Accelerator Progress Report (in press)

[18] Ruffini, R. \& Stella, L. 1983, A\&A, 119, 35

[19] Merafina, M., Ruffini, R. 1989, A\&A, 221, 4

[20] Merafina, M. \& Alberti, G. 2014, Phys. Rev. D, 89, 123010

[21] Bechtol, K., Drlica-Wagner, A., Balbinot, E. et al. 2015, ApJ, 807, 50

[22] Daylan, T., Finkbeiner, D. P., Hooper, D. et al. 2016, Physics of the Dark Universe, 12, 1

[23] Geringer-Sameth, A., Koushiappas, S. M. \& Walker, M. G. 2015, Physical Review D, 91, 083535

[24] Bonnivard, V., Combet, C., Maurin, D. et al. 2015, ApJL, 808, L36

[25] Binney, J. \& Tremaine, S. 2008, Galactyc Dynamics, 2nd edn. Princeton Univ. Press, Princeton, NJ

[26] Charbonnier, A., Combet, C. \& Maurin, D. 2012, Computer Physics Communications, 183, 656

\footnotetext{
${ }^{3}$ https://portal.cta-observatory.org/Pages/Home.aspx
} 
[27] Bonnivard, V., Hütten, M., Nezri, E. et al. 2016, Computer Physics Communications, 200, 336

[28] Walker, M. G., Mateo, M., Olzewski, E. M. et al. 2015, ApJ, 808, 108

[29] Bonnivard, V., Combet, C., Maurin, D. et al. 2015, MNRAS, 446, 3002

[30] Bonnivard, V., Combet, C., Daniel, M. et al. 2015, MNRAS, 453, 849

[31] Einasto, J. 1965, Trudy Astrofizicheskogo Instituta Alma-Ata, 5, 87

[32] Hernquist, L. 1990, ApJ, 356, 359

[33] Zhao, H. 1996, MNRAS, 278, 488

[34] Simon, J. D., Drlica-Wagner, A., Li, T. S. et al. 2015, ApJ, 808, 95

[35] Walker, M. G., Mateo, M., Olzewski, E. W. et al. 2009, AJ, 137, 3109

[36] Hayashi, K., Ichikawa, K., Matsumoto, S. et al. 2016, arXiv e-prints

\section{Discussion}

In the following, we report the questions to the speaker $(\mathrm{Q})$ along with the corresponding answers (A) given during the FRAPWS2016 conference.

(Q) G. AURIEMMA: I am curious to know if you considered the possible interference of this type of DM with nucleosynthesis.

(A) M. MERAFINA: No. In my opinion, at nucleosynthesis time the game is over, and conglomerates do not interact with other baryonic matter, except gravitationally.

(Q) E. GOUVEIA DAL PINO: I have a naïve question. Years ago, there was a search for massive DM - the so-called MACHO searches. The conclusion was that MACHOs would not be enough to explain DM. But you are now proposing an alternative model for massive DM based on strange matter. How do you overcome the MACHO results?

(A) M. MERAFINA: This is a different situation. The masses are very different, and also the possible cosmological origin of strange DM. Now the problem is the detection of gamma rays from products of annihilation of conglomerates of $\Lambda^{*}$. There are only two events and, at the moment, are controversial. We must wait in order to detect other gamma rays as possible proofs of the existence of strange DM.

(Q) M. BUCHER: In the early Universe, at least if one believes the standard BBN scenario, there was a high degree of entropy per baryon, a situation quite unlike nuclear matter in neutron stars, which would be regarded at $T=0$ at the same density. How does this affect the formation of conglomerate in the early Universe?

(A) M. MERAFINA: In no way, because you have to look for the right density conditions. The high density increases only the possibility to form conglomerates, being the distances among $\Lambda^{*}$ particles small and favourable to exchange of $K^{-}$in $\Lambda^{*}$ (like explained in [[1]]). Then strong interactions create the mass defect in order to make stable the conglomerate. 